\section{A repeating rainbow}

In Drosophila embryos, hunchback (hb) messenger RNA is tightly regulated by recruitment of a complex that represses its translation at the posterior of the embryo. Pumilio (Pum) binds hb mRNA through a defined element in its $3^{\prime}$ untranslated region, and then recruits two other repressivefactors, Nanos (Nos) and Brain Tumour (Brat). How is the sequential formation of this complex controlled? Theregion of Pum that is required for this has been narrowed down to theregion that contains Puf repeats, so named after Pum and another protein that contains these repeats, FBF. This critical region is highly conserved in human Pum1, stressing its importance. So how similar are their structures? Very is the answer. The crystal structure of the crucial region of Pum from both is now reported by two groups in Cell and $\mathrm{M}$ olecular Cell; and, in addition to revealing a conserved 'rainbowlike' structure, they reveal some intriguing observations about their similarity to helical-repeat proteins, and the common surface used to mediateinteractions with both proteins and RNA.

Both crystal structures show that Puf repeats align to form an extended, curved molecule. Each repeat is related to thenext by $\sim 20^{\circ}$, leading to a rainbow-like arc structure. Intriguingly, this structure is similar to repeatsfound in the family of helicalrepeat proteins, including $\beta$-catenin and karyopherin- $\alpha$. M oreover, positive charge is concentrated along the inner concavesurface - equival ent to thesurface wheremany helical-repeat proteins show the greatest sequence conservation and interact with their binding partners.

This concentration of charge, together with data from previously characterized mutations in this region, led both groups to propose that the concave surface might provide the binding site for RNA. To investigate this, Edwards and colleagues used mutagenesis to identify

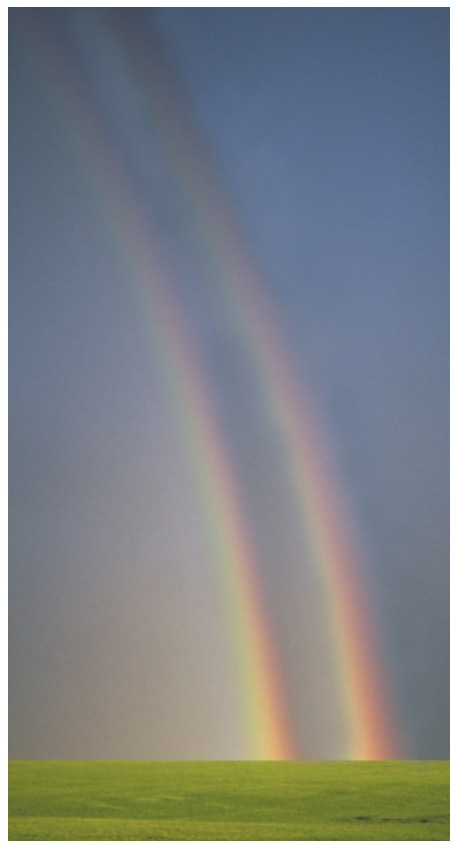

the sites important for binding, and found two lines of evidence showing that the concave surface contacts RNA. Does this reflect a general property of the Puf-domain protein family? From an alignment with other members, Wang and colleagues show that the basic nature of the concave surface is conserved, and predict that it probably has the same function in binding RNA.

Next Edwards and colleagues asked which regions bind the two other members of the complex Nos and Brat. To do this, they tested sets of mutants that bind RNA but cannot interact with one or the other factor. They found that the Pum surface that interacts with Nos includes the eighth repeat and thecarboxy-terminal tail region. Focusing on the site that interacts with Brat, they showed that this is limited to repeats seven, eight and nine. The position of this site relative to theNos-binding region indicates that $\mathrm{N}$ os and Brat might bind cooperatively, and could explain why Brat is recruited only after Nos is present in the complex.

This observation - that Pum binds RNA through its concave surface - indicates that the helicalrepeat family, previously characterized by an extended surface that is proposed to mediate protein-protein interactions, is more versatile, and also uses this surface for protein-RNA interactions.

Alison Schuldt

(2) References and links ORIGINAL RESEARCH PAPERS Edwards, T. A. et al. Structure of Pumilio reveals similarity between RNA and peptide binding motifs. Cell 105, 281-289 (2001) | Wang, X. et al. Crystal structure of a Pumilio homology domain. Mol. Cell 7, 855-865 (2001)

\section{IN BRIEF}

\section{INTRACELLULAR TRANSPORT}

Tuberin-dependent membrane localization of polycystin-1: a functional link between polycystic kidney disease and the TSC2 tumour suppressor gene. Kleymenova, E. et al. Mol. Cell 7, 823-832 (2001)

Mutation of the PKD 1 geneis responsiblefor $\sim 85 \%$ of cases of autosomal dominant polycystic kidney disease. $63 \mathrm{bp}$ upstream of PKD 1 is the tuberous sclerosis 2 (TSC2) tumour-suppressor gene, and a functional link between the PKD 1 gene product polycystin-1 - and the TSC 2 product, tuberin, has now been made. Kleymenova et al. show that, in tuberin-deficient cells, intracellular transport of polycystin-1 is disrupted, implicating tuberin as a determinant in the functional localization of polycystin-1.

\section{EXOCYTOSIS}

SNAREs are concentrated in cholesterol-dependent clusters that define docking and fusion sites for exocytosis.

Lang, T. et al. EMBO J . 20, 2202-2213 (2001)

SNARE proteins are highly enriched in lipid rafts in PC12 cells: implications for the spatial control of exocytosis.

Chamberlain, L. H. et al. Proc. Natl Acad. Sci. USA 98, 5619-5624 (2001)

Pairing of cognate SNARE proteins on vesiclesand target membranes is believed to bethe molecular basis for membrane fusion. It is suspected that several trans-SNARE complexes take part in this interaction, but this has not been documented. Two papersnow show that SNARE proteins are concentrated in cholesterol-rich microdomains on the plasma membrane, and that their association with these domains is important for exocytosis. This supports the concept that SN ARE complexes are organized spatially for efficient membranefusion.

\section{TECHNOLOGY}

Protein quantification from complex protein mixtures using a proteomics methodology with single-cell resolution.

Zhang, H. T. et al. Proc. Natl Acad. Sci. USA 98, 5497-5502 (2001)

Immunodetection amplified by T7 RNA polymerase(IDAT) - a new technique for protein quantification - is at least $10^{9}$ times moresensitive than themost sensitive ELISA technique available today. The technique consists of coupling double-stranded oligonucleotides containing the $T 7$ promoter coval ently to antibodies against a given antigen, amplifying RNA from the oligonucleotides with T7 RNA polymerase and quantifying the radioactive reaction product. Themethod is more sensitive than immuno-PCR, and it is also more accurate owing to thelinearity of the amplification reaction by T7 RNA polymerase. Theauthors suggest that the method has potential as an automated detection system for chip-based proteomics. 